\title{
A Comparative Evaluation of Guttapercha Removal by Various Rotary Files: An in Vitro Study
}

\author{
Anamika Kumari ${ }^{1}$, Ruchi Gupta ${ }^{2}$, Anil K Tomer ${ }^{3}$ \\ ${ }^{1}$ PG Student, ${ }^{2}$ Professor, ${ }^{3}$ Professor and Head, Dept of Conservative Dentistry and Endodontics, DivyaJyoti \\ College of Dental Sciences and Research, Modinagar, Ghaziabad
}

\begin{abstract}
:
Aim: To compare the efficacy of different rotary instruments for removing obturating material from root canals.

Materials and Methods: Thirty extracted human premolar teeth having a single canal were selected. The biomechanical preparation of the root canals was done with Protaperrotary file and obturation was done with corresponding guttapercha cone. The samples were divided into three groups according to the rotary file used for removing the obturating material: Group I - Neoniti; Group II - Protaper D; Group III - Hyflex. The amount of remaining filling material after the retreatment procedure was checked under stereomicroscope. The data was statistically analysed by One way Anova test.
\end{abstract}

Results: Group I(Neoniti) left less remaining filling material compared with group II (Protaper D) and group III (Hyflex).

Conclusion: The Neoniti file was most effective in removing the gutta-percha from the root canal.

Keywords: Neoniti, Retreatment, Hyflex, Protaper D

\section{Introduction}

The principle objective of endodontic therapy is to retain the tooth in proper form and function. But in few cases, patients may report with failure. In such cases, retreatment is the treatment of choice. Complete removal of the root canal content and access to the apical foramen in a retreatment are mandatory for the proper cleaning and reobturation.[1,2] Gutta-percha and endodontic sealer are widely used as filling materials, and their effective removal in endodontic retreatment is considered essential for success.[3,4]

Many techniques have been employed for the removal of gutta-percha (GP) in root-filled teeth. These include endodontic hand files combined with heat or chemical solvents, engine-driven rotary files, ultrasonic instruments, heat-carrying instruments and lasers.[5,6]

ProTaperTM (DentsplyMaillefer, Ballaigues, Switzerland) system exhibits progressively variable tapers of each instrument that develop a "progressive preparation" in both the vertical and horizontal directions. The ProTaper ${ }^{\mathrm{TM}}$ cross-sectional design mimics that of a reamer, with three machined cutting edges and convex core. $[7,8]$

Neoniti (Neolix, Châtres-la-Forêt, France) is a newly introduced NiTi rotary system with a nonhomogeneous rectangular cross section and multiple taper in a single instrument. It consists of one $\mathrm{C} 1$ and three A1 (with \#20, \#25 and \#40 tip sizes) files. The taper in the A1 \#25 file is 0.08 from D0 up to D5; whereas from D5 to D16 the taper is 0.04. It is manufactured using a newly developed wirecut electrical discharge machining (WEDM) process. The manufacturer claims that this manufacturing process is highly precise down to the micron, oil-free and clean and stress is limited to the metal surface during this process. Furthermore, it produces a rough surface, resulting in abrasive properties that enhance the speed of root canal preparation. These files undergo appropriate heat treatment that results in high flexibility and shape memory of this system.[9]

Hyflex ${ }^{\mathrm{TM}} \mathrm{CM}$ nickel-titanium (NiTi) Files (Coltene-Whaledent, Allstetten, Switzerland) is produced by an innovative methodology (patent pending) which uses a unique process that controls the material's memory (a complex heating and cooling treatment).[10]

So far no studies have evaluated the efficacy of Neoniti and Hyflex files for the removal of guttapercha in comparison with Protaper retreatment file. The purpose of this study was to evaluate and analyse the efficacy of ProTaper retreatment files, Neoniti and Hyflex files forremoval of gutta-percha during retreatment.

\section{Material And Methods}

Thirty extractedsingle-rooted human premolars were selected and stored in a $0.1 \%$ thymol. Access cavity preparation was done and working length was determined by inserting a size $10 \mathrm{~K}$ file (Dentsply/Maillefer) into the root canal until it was visible at the apical foramen and subtracting $1 \mathrm{~mm}$ from that length. Root canal preparation was done using ProTaper universal rotary files (DentsplyMaillefer, Switzerland) 
as per manufacturer's instructions. All canals were prepared upto F3ProTaper file. 2.5\% $\mathrm{NaOCl}$ (Avorice, India) was used for irrigation after each instrument. The obturation of root canals was done with ProTaper gutta-percha cones (Dentsply/Maillefer) with AH plus (Dentsply De Trey, Konstanz, Germany) root canal sealer. The teeth were temporarily restored with Cavit-G (3M Espe, Germany), and stored under $100 \%$ humidity at $37{ }^{\circ} \mathrm{C}$. The adequacy of root fillings was confirmed by radiographic examination in buccolingual and mesiodistal direction. Teeth were randomly divided into three experimental groups.

\section{Group 1}

Neoniti C1, an orifice opener followed by Neoniti A1 for guttapercha removal and root-canal preparation to full working length were used. Thespeed and torque used for this file was $350-550 \mathrm{rpm}$ and $1.5 \mathrm{Ncm}$ respectively.

\section{Group 2}

ProTaper Universal retreatment instruments were used to remove the filling material. D1, D2, and D3 were used sequentially applying a crown-down technique until the working length was reached. The instruments were used with an electric motor (X-Smart; DentsplyMaillefer) at a constant speed of $500 \mathrm{rpm}$ for D1, D2, and D3, with a torque of $3 \mathrm{Ncm}$.

\section{Group 3}

Hyflex retreatment rotary file was used to remove the guttapercha.Hyflex $\mathrm{CM}^{\mathrm{TM}}$ instruments were used according to manufacturer's recommendations i.e., 06/20, 06/25, 06/30, 06/35, 06/40.

In order to standardize the procedure, each file was discarded after being used five times. Gutta-percha removal was considered completed when no filling debris was observed either on the instrument flutes or in the irrigating solution. The smoothness of canal walls was checked by tactile sensitivity using the last instrument.

To eliminate inter operator variability, the same operator carried out all intracanal procedures. Assessment of effective gutta-percha removal was done by calculating the obturating material remnants in each tooth at coronal, middle and apical third of the root canal. The percentage area of residual filling material at crosssections within the canals was analyzed using the On Demand 3D App software

\section{Results}

Remnants of filling material were found in all samples regardless of the groups examined. Graph 1 shows the amount of remaining obturating material in three groups. The data was statistically analysed using SPSS version 18.0 software and tested using One Way ANOVA test followed by Bonferroni correction. A p value of 0.05 was considered to be statistically significant. These results demonstrated that group I (Neoniti) left significantly less remaining material compared with group II (Protaper) and group III (Hyflex) $(\mathrm{p}<0.05)$. Maximum amount of remaining filling material was seen with Hyflex rotary file which was also statistically significant. Maximum amount of Gutta-percha was found at coronal third and least in apical third of the root canal in all the groups.

\section{Discussion}

Endodontic retreatment is a procedure performed on a tooth that had received prior attempted definitive treatment resulting in a condition requiring further endodontic treatment to achieve a successful result. The main success of endodontic retreatment relies on the complete removal of root canal filling material in order to regain access to the apical foramen so that it facilitates the sufficient cleaning and shaping of entire root canal system.[2]

Endodontic failure occurs even when the highest standard and the most meticulous treatment procedure is adhered. When conventional root canal treatment fails, endodontic retreatment is the preferred option as it is one of the most conservative methods. Obturating material in failed endodontic cases and necrotic tissue \& bacteria, covered by obturating material, may be responsible for periapical inflammation. As much as possible, the obturating material must be removed to reduce the number of microorganisms within the canal.[4]

Insufficient removal of filling material impairs the removal of necrotic tissue or remnant bacteria in the root canal which leads to a failure. In recent years, the use of nickel-titanium (NiTi) rotary files and automated root canal devices has been increasing in endodontic treatments. The advantages of rotary NiTi instruments over hand instruments include facilitating canal preparation, preserving the shape of curved canals and producing smooth surfaces in lesser time than with manual instruments.[6] The single use of endodontic instruments was recently recommended to decrease instrument fatigue and possible cross contamination, cost effectiveness and reducing the number of NiTi rotary instruments required for canal preparation. The present study compared these new techniques with the ultimate aim of establishing whether they are able to remove filling material from root canals more effectively than other methods. 
Anterior teeth were selected because in these, root canals were usually straight so there were less chances of variations in result while analyzing the efficacy of different rotarytechnique in removing guttapercha.

In this study, none of the three techniques completely removed the filling material from the canal walls in any of the samples. This finding agrees with several previous studies.[7] The results revealed that the Neonitiinstrument (group I) was most effective in removing guttapercha. The better performance of Neoniti instrument may be attributed to their design. The neoniti $\mathrm{C} 1$ file has a high cutting efficiency, no screwing effect, and good flexibility even towards the handle, allowing good tactile perception during the circumferential brushing action. The repositioning of the canal orifices can be achieved easily and quickly.

The neoniti A1 file has no screwing effect. It can achieve an easy and safe access to the apex even in the case of curved canals, and has a rounded gothic tip, achieving a satisfying shape of the apex for later successful root-canal filling.

However, ProTaper retreatment files were found to be unable to render the canals free of root filling material.The Hyflex rotary files (group III) was significantly least effective in removing gutta-percha.

Maximum amount of gutta-percha was found at coronal third and least in apical third of the root canal in all the groups.This might be explained due to more amount of Gutta- percha in the coronal third and the difference between the protapergutta- percha and the taper of the rotary files used to remove gutta - percha. Further research is required to compare the amount of remaining filling material at different levels of root canal using other instrumentation systems.

\section{Conclusion}

Within the parameters of this in vitro study it can be concluded that among all instrumentation techniques used for the removal of filled material in endodontic retreatment, none of the technique was $100 \%$ effective in removing the filling materials, but the Neonitiretreatment file was maximum efficient in comparison to other groups.

\section{References}

[1]. Schirrmeister J, Wrbad KT, Meyer K, Altenburger M, Hellwig E. Efficacy of different rotary instruments for gutta-percha removal in root canal retreatment. J Endod 2006;32:469-472.

[2]. Mollo A, Botti G, Goldoni NP, Randellini E, Paragliola R, Chazine M, Ounsi HF, Grandini S. Efficacy of two Ni-Ti systems and hand files for removing gutta-percha from root canals. IntEndod J 2012;45:1-6.

[3]. Hulsmann M, Bluhm V. Efficacy, cleaning ability and safety of different rotary NiTi instruments in root canal retreatment. IntEndod J 2004;37:468-476.

[4]. Saad AY, Al-Hadlaq SM, Nasser H, Al-Katheeri.Efficacy of two rotary NiTi instruments in the removal of gutta-percha during root canal retreatment. J Endod 2007;33(1):38-41.

[5]. Marfisi K, Mercade M, Plotino G, Duran-Sindreu F, Bueno R, Roig M. Efficacy of three different rotary files to remove guttapercha and Resilon from root canals. IntEndod J 2010;43:1022-1028.

[6]. Unal GC, Kaya B, Tac AG, Kececi AD. A comparison of efficacy of conventional and new retreatment instruments to remove gutta-percha in curved root canals: an ex vivo study. IntEndod J 2009;42:344-350.

[7]. Tasdemir T, Yildirim KET, Celik D. Efficacy of three rotary NiTi instruments in removing gutta-percha from root canals. IntEndod J 2008;41:191-196.

[8]. Somma F, Cammarota G, Plotino G, Grande NM, Pameijer CH. The effectiveness of manual and mechanical instrumentation for the retreatment of three different root canal filling materials. J Endod 2008;34(4):466-469.

[9]. Gergi R, Sabbagh C. Effectiveness of two nickel-titanium rotary instruments and a hand file for removing gutta-percha in severely curved root canals during retreatment: an ex vivo study. IntEndod J 2007;40(7):532-537.

[10]. Surakanti JR, Venkata RCP, Vemisetty HK, Dandolu RK, Jaya NKM, Thota S.Comparative evaluation of apically extruded debris during root canal preparation using ProTaper ${ }^{\mathrm{TM}}$, Hyflex ${ }^{\mathrm{TM}}$ and Waveone ${ }^{\mathrm{TM}}$ rotary systems.J Conserv Dent 2014; 17(2): 129-132.

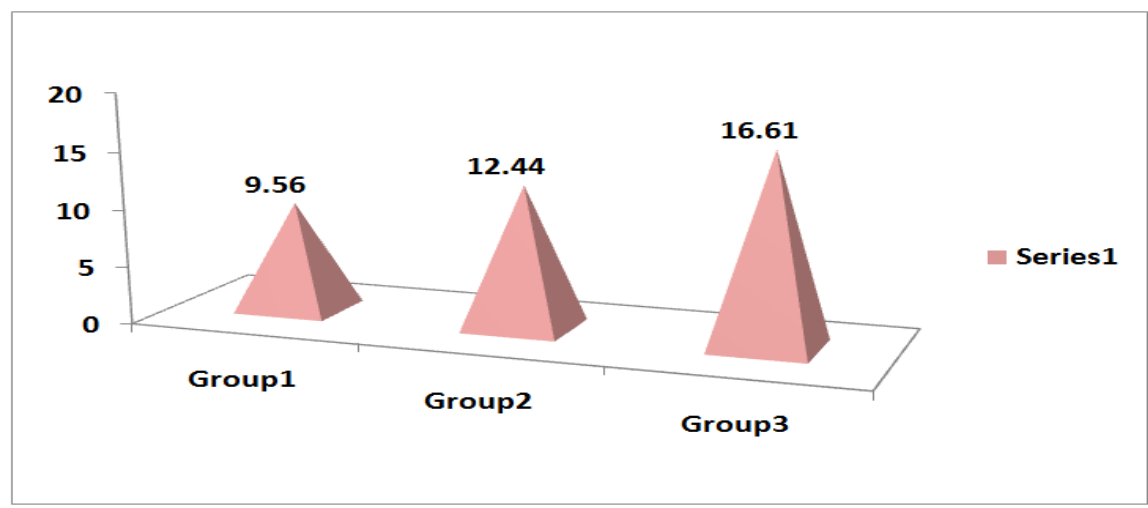

Graph 1: Graphical Representation Showing Remaining Gutta-Percha Left in the Canal 